Research Article

\title{
Chaotic Behaviors of Symbolic Dynamics about Rule 58 in Cellular Automata
}

\author{
Yangjun Pei, ${ }^{1}$ Qi Han, ${ }^{1}$ Chao Liu, ${ }^{1}$ Dedong Tang, ${ }^{1}$ and Junjian Huang ${ }^{2}$ \\ ${ }^{1}$ School of Electrical and Information Engineering, Chongqing University of Science and Technology, Chongqing 401331, China \\ ${ }^{2}$ Department of Mathematics and Information Engineering, Chongqing University of Education College, Chongqing 400065, China
}

Correspondence should be addressed to Qi Han; hanqicq@163.com

Received 14 April 2014; Accepted 25 June 2014; Published 22 July 2014

Academic Editor: Chuandong Li

Copyright (C) 2014 Yangjun Pei et al. This is an open access article distributed under the Creative Commons Attribution License, which permits unrestricted use, distribution, and reproduction in any medium, provided the original work is properly cited.

The complex dynamical behaviors of rule 58 in cellular automata are investigated from the viewpoint of symbolic dynamics. The rule is Bernoulli $\sigma_{\tau}$-shift rule, which is members of Wolfram's class II, and it was said to be simple as periodic before. It is worthwhile to study dynamical behaviors of rule 58 and whether it possesses chaotic attractors or not. It is shown that there exist two Bernoullimeasure attractors of rule 58. The dynamical properties of topological entropy and topological mixing of rule 58 are exploited on these two subsystems. According to corresponding strongly connected graph of transition matrices of determinative block systems, we divide determinative block systems into two subsets. In addition, it is shown that rule 58 possesses rich and complicated dynamical behaviors in the space of bi-infinite sequences. Furthermore, we prove that four rules of global equivalence class $\varepsilon_{4}^{3}$ of CA are topologically conjugate. We use diagrams to explain the attractors of rule 58, where characteristic function is used to describe that some points fall into Bernoulli-shift map after several times iterations, and we find that these attractors are not global attractors. The Lameray diagram is used to show clearly the iterative process of an attractor.

\section{Introduction}

Cellular automaton (CA) was first introduced in 1951 [1]. $\mathrm{CA}$ is a mathematical model consisting of large numbers of simple identical components with local interactions [2]. The simple components act together to produce complex global behavior. CA performs complex computation with high degree of efficiency and robustness. Three major factors have resulted in the revival of interest in the behavior of cellular systems [3]. First, the development of powerful computers and microprocessors has made the rapid simulation of CA possible. Second, the use of CA to simulate physical systems has attracted much interest in the scientific community. Third, the advent of VLSI as an implementation medium has focused attention on the communication requirements of successful hardware algorithms. In recent years, many applications of CA have been reported, especially in cryptography [4-9], image processing [10, 11], and associative memory $[12,13]$.

In recent years, many researches were devoted to find properties of rules of CA, especially binary one-dimensional
CA. Because the rules of binary one-dimensional CA are simple to study, the evolutions of these rules can be reflected directly by image. In 1980s, Wolfram proposed CA as models for physical systems which exhibit complex or even chaotic behaviors based on empirical observations, and he divided the 256 ECA (binary one-dimensional CA with radius 1) rules informally into four classes using dynamical concepts like periodicity, stability, and chaos [14-16]. Recently, [1722] focused on ECA in detail. In [17], Chua et al. listed 256 Boolean function "cubes" defining all Boolean functions of three binary variables, and they elucidated that every binary cellular automata of any spatial dimension was a special case of a cellular automaton with the same neighborhood size. In [18], Chua et al. partitioned the entire set of 256 local rules into 16 different gene families. Chua et al. [19] mentioned that each rule has three globally equivalent local rules determined by three corresponding global transformations, namely, leftright transformation $T^{\dagger}$, global complementation $\bar{T}$, and left-right complementation $T^{*}$. Each equivalence class is identified by $\varepsilon_{m}^{\kappa}$, where $\kappa$ is complexity index and $m$ is index of $\kappa$ th class. In [20], the authors presented that 112 rules of 
256 local rules were Bernoulli $\sigma_{\tau}$-shift rules. Each of the 112 Bernoulli $\sigma_{\tau}$-shift rules has an ID code $B_{N}[\alpha, \beta, \tau]$, where $\alpha$ denotes the number of attractors of rule $N, \beta$ denotes the slope of the Bernoulli $\sigma_{\tau}$-shift map, and $\tau$ denotes the relevant forward time- $\tau$. Hence, the space-time evolution of any one of the 112 rules on their attractors can be uniquely predicted by two parameters: $\beta= \pm 2^{\sigma}$ and $\tau$. For example, rule 58 has two attractors $(\beta=2, \sigma=1, \tau=1)$ and $(\beta=1 / 2, \sigma=-1, \tau=2)$. Wolfram considered that Bernoulli $\sigma_{\tau}$-shift rules were simple as periodic, but he did not find other complex dynamical behaviors. Recently, some authors [23-28] found that some rules of Bernoulli $\sigma_{\tau}$-shift rules are chaotic in the sense of both Li-York and Devaney. However, they did not involve and studied the dynamical behavior of rule 58. For 256 rules, every rule has its properties which are different from other rules. So, we need to study these special properties for every rule for practical application.

In this paper, the complex dynamical behavior of rule 58 in cellular automata is studied in detail. It will be shown that rule 58 with bi-infinite bit strings possesses rich and complicated dynamical behaviors. The rest of the paper is organized as follows. In Section 2, the basic concepts of onedimension CA (1DCA) and symbolic dynamics are introduced. The Boolean functions of rule 58 are also presented, and expressions of two attractors are given. In Section 3, two subsystems of rule 58 are characterized. We prove that rule 58 is topologically mixing and chaotic in the sense of LiYork and Devaney on the $\Lambda_{\lambda_{1}^{58}}$ and $\Lambda_{2}^{58}$, respectively. We also prove that four rules of global equivalence class $\varepsilon_{4}^{3}$ of CA are topologically conjugate. In Section 4, characteristic function is used to describe that all points of rule 58 fall into Bernoulli-shift map after several times iterations, and the Lameray diagram is used to show clearly the iterative process of an attractor. We mention that rule 58 can be used in associative memory. Section 5 presents some conclusions.

\section{Preliminaries}

For simplicity, for some notations about symbolic dynamics, one can refer to [27-29].

It follows from [30] that the Boolean function of rule 58 is

$$
\left[f_{58}(x)\right]_{i}=x_{i-1} \cdot \bar{x}_{i} \oplus \bar{x}_{i-1} \cdot x_{i+1}
$$

$\forall x \in S^{Z}, i \in Z$, where “." “-," and “ $\oplus$ ” stand for "AND," "NOT," and "XOR" logical operation, respectively. Sometimes, "." is omitted for simplicity. The truth table of Boolean functions of rule 58 is shown in Table 1. The subsets, denoted by $\Lambda_{1}^{58}$ and $\Lambda_{2}^{58}$, are derived from the parameters of rules 58: $\beta=2, \sigma=1$, and $\tau=1$ and $\beta=1 / 2, \sigma=-1$, and $\tau=2$, respectively; that is,

$$
\begin{aligned}
& \Lambda_{1}^{58}=\left\{x \in S^{Z} \mid\left[f_{58}(x)\right]_{i}=x_{i+1}, \forall i \in Z\right\}, \\
& \Lambda_{2}^{58}=\left\{x \in S^{Z} \mid\left[f_{58}^{2}(x)\right]_{i}=x_{i-1}, \forall i \in Z\right\},
\end{aligned}
$$

where $f^{2}(\cdot)$ denotes two iterations for a bit.

The subsets $\Lambda_{1}^{58}$ and $\Lambda_{2}^{58}$ will be rigorously characterized from the viewpoint of symbolic dynamics.
TABLE 1: The truth table of Boolean function of rules 58.

\begin{tabular}{lc}
\hline$x_{i-1} x_{i} x_{i+1}$ & $x_{i-1} \bar{x}_{i} \oplus \bar{x}_{i-1} x_{i+1}$ \\
\hline 000 & 0 \\
001 & 1 \\
010 & 0 \\
011 & 1 \\
100 & 1 \\
101 & 1 \\
110 & 0 \\
111 & 0 \\
\hline
\end{tabular}

\section{Dynamical Behaviors of $f_{58}$ on Two Subsystems}

In this section, dynamical behaviors of $f_{58}$ will be researched. We find that rule 58 is chaotic in the sense of Li-York and Devaney on its two subsystems.

Proposition 1. For rule 58, there exists a subset $\Lambda_{1}^{58} \subset S^{Z}$ which satisfies $\left.f_{58}\right|_{\Lambda_{1}^{58}}=\left.v\right|_{\Lambda_{1}^{58}}$ if and only if $\forall x=\left(\ldots, x_{-1}\right.$, $\left.x_{0}, x_{1}, \ldots\right) \in \Lambda_{1}^{58} ; x_{i-1}, x_{i}$ and $x_{i+1}$ have the following relations:

(i) if $x_{i}=1$, then $x_{i-2}=1, x_{i-1}=0, x_{i+1}=0$, and $x_{i+2}=$ $1 ; x_{i-2}=0, x_{i-1}=0, x_{i+1}=1$, and $x_{i+2}=0 ; x_{i-2}=0$, $x_{i-1}=1, x_{i+1}=0$, and $x_{i+2}=1$;

(ii) if $x_{i}=0$, then $x_{i-2}=0, x_{i-1}=0, x_{i+1}=0, x_{i+2}=0$, $x_{i-1}=1$, and $x_{i+1}=1$.

The explicit proof of this proposition is presented in the Appendix.

Remark 2. From the definition of subsystem, we know that $\left(\Lambda_{1}^{58}, f_{58}\right)$ is subsystems of $\left(S^{Z}, f_{58}\right)$.

The dynamical behaviors of $f_{58}(x)$ on the set $\Lambda_{1}^{58}$ are shown as follows.

Let $P_{1}^{58}=\left\{r_{0}, r_{1}, r_{2}, r_{3}, r_{4}\right\}$ be a new state set, where $r_{0}=$ (000), $r_{1}=(010), r_{2}=$ (011), $r_{3}=(101), r_{4}=(110)$, and $\omega_{1}^{58}=\left\{\left(r r^{\prime}\right) \mid r=\left(b_{0} b_{1} b_{2}\right), r^{\prime}=\left(b_{0}^{\prime} b_{1}^{\prime} b_{2}^{\prime}\right) \in P_{1}^{58}, \forall 1 \leq j \leq 2\right.$ such that $\left.b_{j}=b_{j-1}^{\prime}\right\}$. Furthermore, subshift $\Lambda_{\omega_{1}^{58}}$ of $v$ is defined as $\Lambda_{\omega_{1}^{58}}=\left\{r=\left(\ldots r_{-1}, r_{0}, r_{1} \ldots\right) \in P_{1}^{58^{Z}} \mid r_{i} \in P_{1}^{58}, r_{i} r_{i+1} \in\right.$ $\left.\omega_{1}^{58}, \forall i \in Z\right\}$. The transition matrix $B_{1}^{58}$ of the $\left.v\right|_{\Lambda_{\omega_{1}^{58}}}$ is

$$
B_{1}^{58}=\left[\begin{array}{lllll}
1 & 0 & 0 & 0 & 0 \\
0 & 0 & 0 & 1 & 0 \\
0 & 0 & 0 & 0 & 1 \\
0 & 1 & 1 & 0 & 0 \\
0 & 0 & 0 & 1 & 0
\end{array}\right] \text {. }
$$

In order to give our results, in the following, some definitions need be introduced.

Definition 3 (see [31]). A square $\{0,1\}$ matrix $A$ is irreducible, if for every pair of indices $i$ and $j$ there is an $n$ such that $A_{i j}^{n}>$ 0 .

Definition 4 (see [31]). A square $\{0,1\}$ matrix $A$ is aperiodic, if there exists $N$, such that $A_{i j}^{n}>0$ and $n>N, \forall i, j$. 


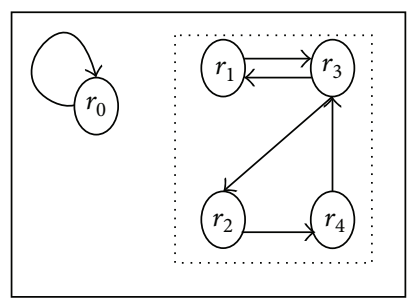

FIgURE 1: The corresponding graph $G_{58}^{1}$ of the matrix $B_{1}^{58}$.

Definition 5 (see [31]). Suppose that $g: X \rightarrow Y$ is a continuous mapping, where $X$ is a compact topological space. $g$ is said to be topologically mixing if, for any two open sets $U, V \subset X, \exists N>0$, such that $g^{n}(U) \cap V \neq \emptyset, \forall n \geq N$.

Definition 6 (see [24]). Let $(X, f)$ and $(Y, g)$ be compact spaces, one says that $f$ and $g$ are topologically conjugate if there is homeomorphism $h: X \rightarrow Y$, such that $h \circ f=g \circ h$.

A square $\{0,1\}$ matrix corresponds to a directed graph. The vertices of the graph are the indices for the rows and columns of $A$. There is an edge from vertex $i$ to vertex $j$ if $A_{i j}=1$. A square $\{0,1\}$ matrix is irreducible if and only if the corresponding graph is strongly connected. If $\Lambda_{A}$ is a twoorder subshift of finite type, then it is topologically mixing if and only if $A$ is irreducible and aperiodic [31].

We give corresponding graph $G_{58}^{1}$ of the matrix $B_{1}^{58}$ in Figure 1. We find that $G_{58}^{1}$ is not a strongly connected graph. Therefore, $\left.f_{58}\right|_{\Lambda_{1}^{58}}$ is not topologically mixing. But the subgraphs $r_{0}$ and $r_{1}, r_{2}, r_{3}$, and $r_{4}$ are strongly connected graph, respectively. So, we can divide $P_{1}^{58}$ into two subsets: $\hbar_{1}^{58}=\left\{r_{0}\right\}$ and $\lambda_{1}^{58}=\left\{r_{1}, r_{2}, r_{3}, r_{4}\right\}$.

Let $h_{1}^{58}=\left\{\left(r r^{\prime}\right) \mid r=\left(b_{0} b_{1} b_{2}\right), r^{\prime}=\left(b_{0}^{\prime} b_{1}^{\prime} b_{2}^{\prime}\right) \in P_{1}^{58}, \forall 1 \leq\right.$ $j \leq 2$, such that $\left.b_{j}=b_{j-1}^{\prime}\right\}$ corresponding to $\hbar_{1}^{58}$ and $\lambda_{1}^{58}=$ $\left\{\left(r r^{\prime}\right) \mid r=\left(b_{0} b_{1} b_{2}\right), r^{\prime}=\left(b_{0}^{\prime} b_{1}^{\prime} b_{2}^{\prime}\right) \in P_{1}^{58}, \forall 1 \leq j \leq 2\right.$, such that $\left.b_{j}=b_{j-1}^{\prime}\right\}$ corresponding to $\lambda_{1}^{58}$. We can obtain the transition matrix $C_{1}^{58}$ corresponding to $\left.v\right|_{\Lambda_{\hbar_{1}^{58}}}$ and transition matrix $D_{1}^{58}$ corresponding to $\left.v\right|_{\Lambda_{\lambda_{1}^{58}}}$, where

$$
C_{1}^{58}=[1], \quad D_{1}^{58}=\left[\begin{array}{llll}
0 & 0 & 1 & 0 \\
0 & 0 & 0 & 1 \\
1 & 1 & 0 & 0 \\
0 & 0 & 1 & 0
\end{array}\right] \text {. }
$$

Based on the above definition and analysis, we give the following results.

Proposition 7. Consider the following:

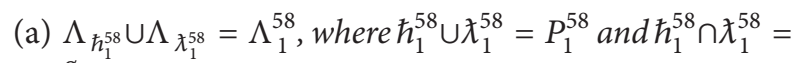
$\emptyset$.

(b) $v: \Lambda_{\lambda_{1}^{58}} \rightarrow \Lambda_{\lambda_{1}^{58}}$ and $v: \Lambda_{\lambda_{1}^{58}} \rightarrow \Lambda_{\lambda_{1}^{58}}$ are topologically conjugate.

(c) $v: \Lambda_{\lambda_{1}^{58}} \rightarrow \Lambda_{\lambda_{1}^{58}}$ is topologically mixing.

(d) $f_{58}: \Lambda_{\lambda_{1}^{58}} \rightarrow \Lambda_{\lambda_{1}^{58}}$ is topologically mixing. (e) The topological entropy ent $\left(\left.f_{58}\right|_{\Lambda_{\lambda_{1}^{58}}}\right)=\operatorname{ent}\left(\left.v\right|_{\Lambda_{\lambda_{1}^{58}}}\right)=$ 0.2812 .

Proof. (a) Let $x=\left\{\ldots, x_{-1}, \stackrel{*}{x}_{0}, x_{1}\right\}$. It is obvious that if $w^{\prime} \prec x$, $w^{\prime} \in \hbar_{1}^{58}$, then $\forall w^{\prime \prime} \in \lambda_{1}^{58}, w^{\prime \prime} \nless x$, thus $x \in \Lambda_{\hbar_{1}^{58}}$; conversely, if $w^{\prime} \prec x, w^{\prime} \in \lambda_{1}^{58}$, then $\forall w^{\prime \prime} \in \hbar_{1}^{58}, w^{\prime \prime} k x$, thus $x \in \Lambda_{\lambda_{1}^{58}}$; namely, $\forall x \in \Lambda_{1}^{58}, x \in \Lambda_{\hbar_{1}^{58}}$, or $x \in \Lambda_{\lambda_{1}^{58}}$. Hence, $\Lambda_{\hbar_{1}^{58}} \cup \Lambda_{\lambda_{1}^{58}}=$ $\Lambda_{1}^{58}$.

The proofs of (b), (c), (d), and (e) can be referred to in Proposition 2 in [28].

Theorem 8. $f_{58}$ is chaotic in the sense of both Li-Yorke and Devaney on $\Lambda_{\lambda_{1}^{58}}$.

Proof. It follows from [32] that the positive topological entropy implies chaos in the sense of Li-Yorke, and topological mixing implies chaos in the sense of Li-Yorke and Devaney, since rule $N=58$ possesses very rich and complicated dynamical properties on $\Lambda_{\lambda_{1}^{58}}$.

Remark 9. Though, $\forall n>0,\left(C_{1}^{58}\right)^{n}>0$, we cannot believe that $\left.f_{58}\right|_{\hbar_{1}^{58}}$ is topologically mixing. Because $C_{1}^{58}$ is not a square $\{0,1\}$ matrix. The topological entropy $\operatorname{ent}\left(\left.f_{58}\right|_{\Lambda_{\hbar_{1}^{58}}}\right)=0$. So, $f_{58}$ is not chaotic in the sense of both Li-Yorke and Devaney on $\Lambda_{\hbar_{1}^{58}}$.

Remark 10. Carefully observing Figure 1, we find that there are several strongly connected subgraphs: $r_{2} \rightarrow r_{3} \rightarrow r_{4} \rightarrow$ $r_{2}, r_{1} \rightarrow r_{3} \rightarrow r_{1}, r_{1} \rightarrow r_{3} \rightarrow r_{2} \rightarrow r_{4} \rightarrow r_{3} \rightarrow r_{1}$, and $r_{0} \rightarrow r_{0}$. The elements of $\Lambda_{1}^{58}$ are composed by all vertices of those strongly connected subgraphs, respectively. For example, $x \in \Lambda_{1}^{58}$ and $x$ is composed of vertices of subgraph $r_{2} \rightarrow r_{3} \rightarrow r_{4} \rightarrow r_{2}$; then we have $r_{0} \nless x$ and $r_{1} \nless x$, and all vertices of the subgraph will appear in $x$, if $|x|=3 k, k=1,2, \ldots$

Proposition 11. For rule 58, there exists a subset $\Lambda_{2}^{58} \mathrm{C}$ $S^{Z}$ which satisfies $\left.f_{58}^{2}\right|_{\Lambda_{2}^{58}}=\left.\varsigma\right|_{\Lambda_{2}^{58}}$ if and only if, $\forall x=$ $\left(\ldots, x_{-1}, x_{0}, x_{1}, \ldots\right) \in \Lambda_{2}^{58}, x_{i-1} x_{i} x_{i+1}$ cannot equal to 010 , $\forall i \in Z$.

Proof. The global map of rule 58 is $\left[f_{58}(x)\right]_{i}=x_{i-1} \bar{x}_{i} \oplus$ $\bar{x}_{i-1} x_{i+1}$, so

$$
\begin{aligned}
{\left[f_{58}^{2}(x)\right]_{i}=} & {\left[f_{58}(x)\right]_{i-1} \cdot \overline{\left[f_{58}(x)\right]_{i}} \oplus \overline{\left[f_{58}(x)\right]_{i-1}} \cdot\left[f_{58}(x)\right]_{i+1} } \\
= & {\left[\left(x_{i-2} \bar{x}_{i-1} \oplus \bar{x}_{i-2} x_{i}\right) \cdot \overline{x_{i-1} \bar{x}_{i} \oplus \bar{x}_{i-1} x_{i+1}}\right] } \\
& \oplus\left[\overline{x_{i-2} \bar{x}_{i-1} \oplus \bar{x}_{i-2} x_{i}} \cdot\left(x_{i} \bar{x}_{i+1} \oplus \bar{x}_{i} x_{i+2}\right)\right] .
\end{aligned}
$$

Then, the proof is similar to proof of necessity and sufficiency in Proposition 1, so the details are omitted.

Let $P_{2}^{58}=\left\{r_{0}, r_{1}, r_{2}, r_{3}, r_{4}, r_{5}, r_{6}\right\}$, where $r_{0}=(000), r_{1}=$ (001), $r_{2}=(011), r_{3}=(100), r_{4}=(101), r_{5}=(110)$, and $r_{6}=(111)$. 


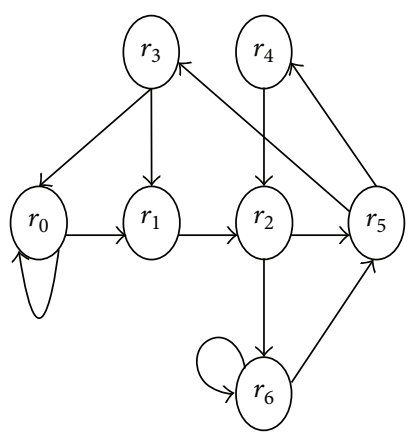

Figure 2: The corresponding graph $G_{58}^{2}$ of the matrix $B_{2}^{58}$.

Remark 12. The transition matrix of subshift $\left(\Lambda_{2}^{58}, \varsigma\right)$ is

$$
B_{2}^{58}=\left[\begin{array}{lllllll}
1 & 1 & 0 & 0 & 0 & 0 & 0 \\
0 & 0 & 1 & 0 & 0 & 0 & 0 \\
0 & 0 & 0 & 0 & 0 & 1 & 1 \\
1 & 1 & 0 & 0 & 0 & 0 & 0 \\
0 & 0 & 1 & 0 & 0 & 0 & 0 \\
0 & 0 & 0 & 1 & 1 & 0 & 0 \\
0 & 0 & 0 & 0 & 0 & 1 & 1
\end{array}\right]
$$

We give corresponding graph $G_{58}^{2}$ of the matrix $B_{2}^{58}$ in Figure 2. It is obvious that $G_{58}^{2}$ is a strongly connected graph. So, $B_{2}^{58}$ is irreducible.

Based on the above analysis, we have the following results.

Proposition 13. Consider the following:

(a) $\left.\varsigma\right|_{\Lambda_{2}^{58}}$ is topologically mixing.

(b) $\left.f_{58}^{2}\right|_{\Lambda_{2}^{58}}$ is topologically mixing.

(c) The topological entropy ent $\left(\left.f_{58}^{2}\right|_{\Lambda_{2}^{58}}\right)=\operatorname{ent}\left(\left.\varsigma\right|_{\Lambda_{2}^{58}}\right)=$ 0.5624 .

Proof. (a) Because $\left(B_{2}^{58}\right)^{n}>0, \forall n \geq 4$, the transition matrix $B_{2}^{58}$ of subshift of finite type $\varsigma$ is irreducible and aperiodic. By $[32,33],\left.\varsigma\right|_{\Lambda_{2}^{58}}$ is topologically mixing.

Theorem 14. $f_{58}^{2}$ is chaotic in the sense of both Li-Yorke and Devaney on $\Lambda_{2}^{58}$.

Proof. It follows from [32] that topological mixing implies chaos in the sense of Li-Yorke and Devaney.

Proposition 15. Consider $f(x) \in \Lambda_{2}^{58}, \forall x \in \Lambda_{2}^{58}$.

Proof. We check that $x_{1}, x_{2} \in \Lambda_{2}^{58}$, where $x_{1}=\left\{\ldots, a_{-2}, a_{-1}\right.$, $\left.a_{0}, a_{1}, a_{2}, \ldots\right\}$. Let $f_{58}^{2}\left(x_{1}\right)=x_{2}$. Suppose that $f_{58}\left(x_{1}\right)=y_{1}$ and $f_{58}\left(x_{2}\right)=y_{2}$, where $y_{1}=\left\{\ldots, b_{-2}, b_{-1}, b_{0}, b_{1}, b_{2}, \ldots\right\}$ and $y_{2}=$ $\left\{\ldots c_{-3}, c_{-2}, c_{-1}, c_{0}, c_{1}, \ldots\right\}$. Table 2 shows the iterative process of bit string $x_{1}$. Observing Table 2 , we have $f_{58}\left(a_{-1} a_{0} a_{1}\right)=$ $b_{0}=c_{0}, \ldots, f_{58}\left(a_{i-1} a_{i} a_{i+1}\right)=b_{i}=c_{i}, \ldots$. So, we get $f_{58}^{2}\left(y_{1}\right)=$ $y_{2}$. Therefore, $y_{1}, y_{2} \in \Lambda_{2}^{58}$. Hence, $f(x) \in \Lambda_{2}^{58}, \forall x \in \Lambda_{2}^{58}$.
TABLE 2: The iterative process of bit string $x_{1}$.

\begin{tabular}{cccccccc}
\hline$x_{1}$ & $\cdots$ & $a_{-2}$ & $a_{-1}$ & $a_{0}$ & $a_{1}$ & $a_{2}$ & $\cdots$ \\
$y_{1}$ & $\ldots$ & $b_{-2}$ & $b_{-1}$ & $b_{0}$ & $b_{1}$ & $b_{2}$ & $\ldots$ \\
$x_{2}$ & $\ldots$ & $a_{-3}$ & $a_{-2}$ & $a_{-1}$ & $a_{0}$ & $a_{1}$ & $\ldots$ \\
$y_{2}$ & $\cdots$ & $c_{-3}$ & $c_{-2}$ & $c_{-1}$ & $c_{0}$ & $c_{1}$ & $\cdots$ \\
\hline
\end{tabular}

Corollary 16. Consider $\left.f_{58}^{2}\right|_{\Lambda_{2}^{58}}=\left(\left.f_{58}\right|_{\Lambda_{2}^{58}}\right)^{2}$.

Proposition 17. Consider the following:

(a) $\left.f_{58}\right|_{\Lambda_{2}^{58}}$ is topologically mixing.

(b) The topological entropy ent $\left(\left.f_{58}\right|_{\Lambda_{2}^{58}}\right)=0.2812$.

The explicit proof of this proposition is presented in the Appendix.

Theorem 18. $f_{58}$ is chaotic in the sense of both Li-Yorke and Devaney on $\Lambda_{2}^{58}$.

Remark 19. It is obviously that $\left(\Lambda_{2}^{58}, f_{58}\right)$ are subsystems of $\left(S^{Z}, f_{58}\right)$. Hence, there are two subsystems for $\left(S^{Z}, f_{58}\right)$.

Next, we will discuss the relationship on four rules of global equivalence class $\varepsilon_{4}^{3}$.

Remark 20. From [20, 28-30], the following results can be obtained:

(1) $f_{58}: S^{Z} \rightarrow S^{Z}$ and $f_{114}: S^{Z} \rightarrow S^{Z}$ are topologically conjugate;

(2) $f_{58}: S^{Z} \rightarrow S^{Z}$ and $f_{163}: S^{Z} \rightarrow S^{Z}$ are topologically conjugate;

(3) $f_{58}: S^{Z} \rightarrow S^{Z}$ and $f_{177}: S^{Z} \rightarrow S^{Z}$ are topologically conjugate.

Remark 21. $f_{58}, f_{114}, f_{163}$, and $f_{177}$ are topologically conjugate, respectively. Therefore, if we know that one of four rules is chaotic in the sense of both Li-Yorke and Devaney in its attractors, we can deem that others of four rules are chaotic in the sense of both Li-Yorke and Devaney in their attractors, respectively. The phenomenon also presents that the global equivalence class introduced by Chua et al. [22] is useful and important for research of rule of cellular automata.

\section{Using Diagrams to Explain Attractors of Four Rules}

From a definition on global characteristic function in [20], the Boolean string $x$ can be associated with a real number $0 . x_{0} x_{1} \ldots x_{I-1} x_{I}$ on the unit interval $[0,1]$ :

$$
\begin{array}{r}
x=\left[x_{0} x_{1} \ldots x_{I-1} x_{I}\right] \longmapsto \phi \triangleq 0 . x_{0} x_{1} \ldots x_{I-1} x_{I}, \\
x_{i} \in\{0,1\},
\end{array}
$$




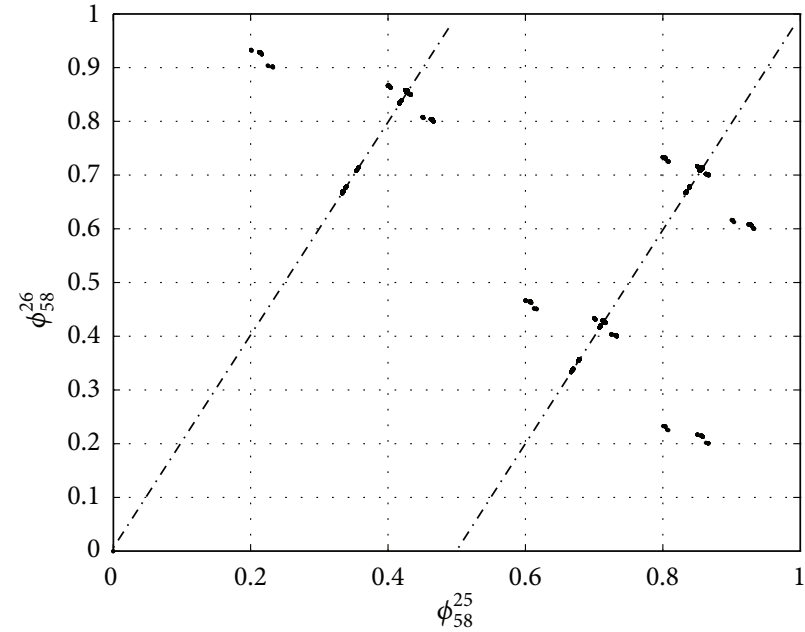

(a)

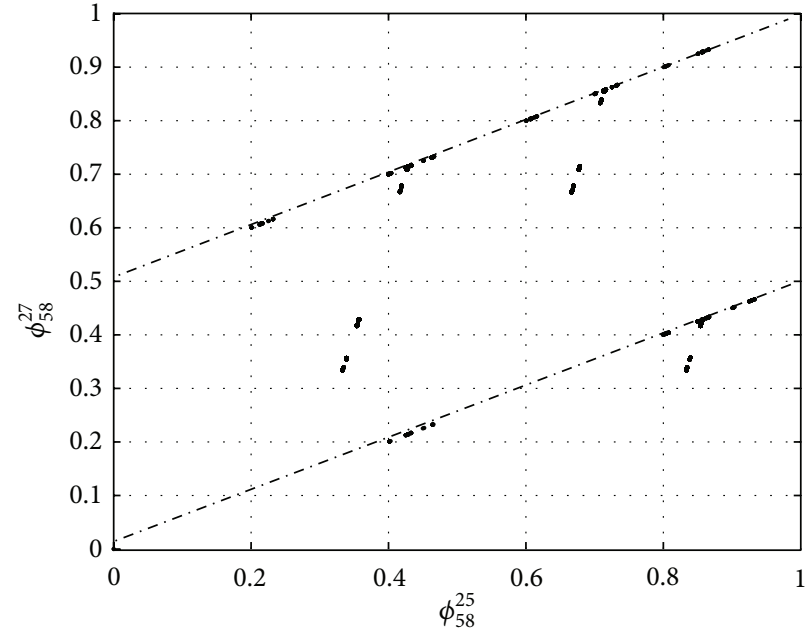

(b)

FIGURE 3: All points fall into Bernoulli-shift map after several iterations under rule 58, where $I=13$.

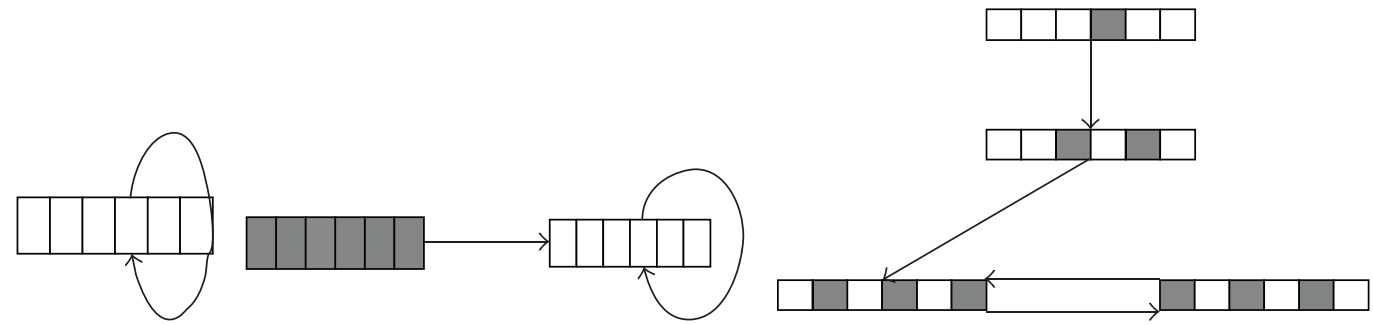

(a)

(b)

(c)

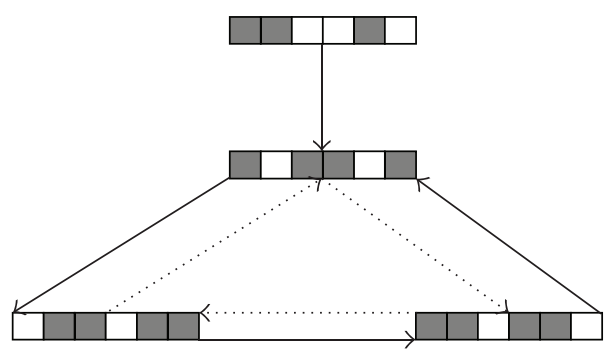

(d)

FIgURE 4: Some attractors of rule 58, where $I=5$, and the white lattice stands for 0 and black for 1 .

where $\phi=\sum_{i=0}^{I} 2^{-(i+1)} x_{i}$ is the decimal form of Boolean string $x=\left[x_{0} x_{1} \ldots x_{I-1} x_{I}\right]$. The CAs' characteristic function $\chi_{N}$ of rule $N$ is defined as

$$
\begin{aligned}
& \chi_{N}: Q[0,1] \longrightarrow Q[0,1], \\
& \text { that is, } \phi_{n}=\chi_{N}^{1}\left(\phi_{n-1}\right), \quad \phi_{n}=\chi_{N}^{2}\left(\phi_{n-2}\right),
\end{aligned}
$$

where $Q$ denotes rational numbers.

Let $I=13$. Figure 3 shows characteristic functions of rule 58. Figure 3(a) describes that some points of rule 58 fall into Bernoulli-shift map after twenty-six iterations, respectively, where $\tau=1$. Figure 3(b) describe that some points of four rules fall into Bernoulli-shift map after twenty-seven iterations, respectively, where $\tau=2$.
If we choose different values of $I$ for the four rules, we can get different initial binary configuration for the evolution of four rules. The different initial binary configuration may lead to different attractor periods. If the value of $I$ is fixed, we find that the attractor period may be different. Let $I=5$; then we can obtain Figure 4, which shows some attractors of rule 58. Figures 4(a) and 4(b) show that the period of attractor is 1 , and the attractor belongs to $\Lambda_{\hbar_{1}^{58}}$ and $\Lambda_{2}^{58}$; Figure 4(c) shows that the period of attractor is 2 , and the attractor belongs to $\Lambda_{\lambda_{1}^{58}} ;$ Figure 4 (d) shows that the attractor belongs to both $\Lambda_{\lambda_{1}^{58}}$ and $\Lambda_{2}^{58}$, where the solid lines represent that the attractor belongs to $\Lambda_{\lambda_{1}^{58}}$ and the dotted lines represent that the attractor belongs to $\Lambda_{2}^{58}$. Let $I=4$; then we can obtain 


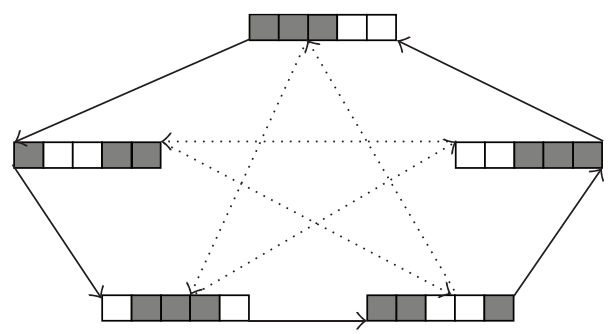

FIgURE 5: Period-5 isle of Eden is shown, where $I=4$, and the white lattice stands for 0 and black for 1.

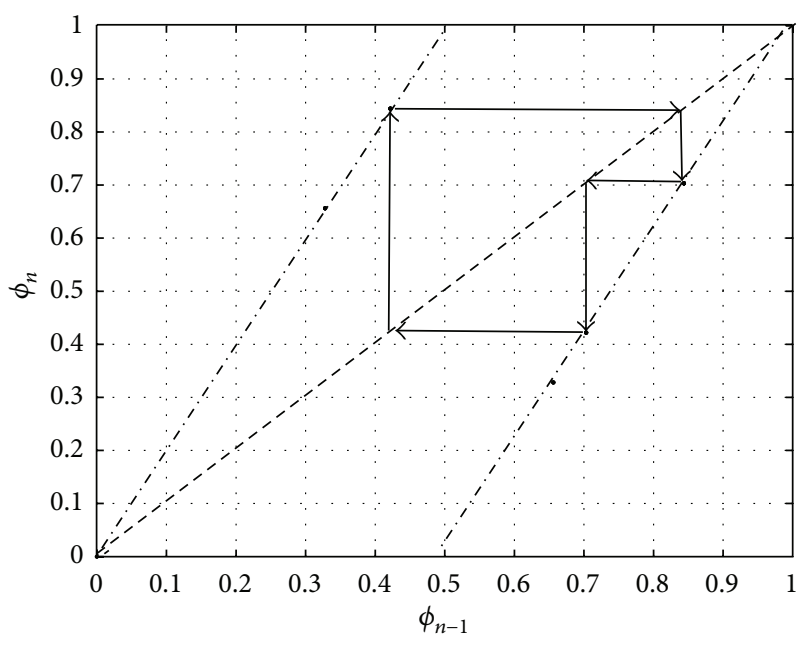

(a)

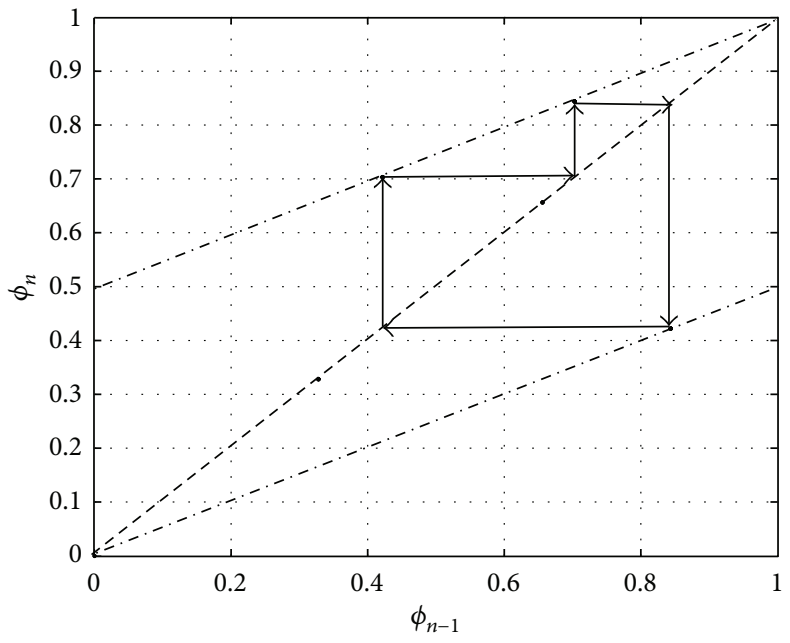

(b)

FIGURE 6: The evolution of characteristic function of the period-3 attractor, where the values of characteristic function of the attractor are $0.7031,0.4219$, and 0.8438 , respectively. (a) shows the iterative process of an attractor, where $\tau=1$, and (b) shows the iterative process of an attractor, where $\tau=2$.

Figure 5, where the solid lines stand for the real evolution of binary configuration 11100, and the dotted lines stand for the attractor belonging to $\Lambda_{2}^{58}$.

Next, we use the Lameray diagram [33] to present our attractors. The diagrams show clearly the iterative process of attractors. In terms of the attractor of Figure 4(d), we get that the values of characteristic function of the attractor are $0.7031,0.4219$, and 0.8438 , respectively. Figure $6($ a) shows the iterative process of an attractor, where $\tau=1$, and Figure 6(b) shows the iterative process of an attractor, where $\tau=2$. Then, we can associate the period-3 attractor of rule 58 in Figure 6(a) as a period-3 point of a continuous map $f:[0,1] \rightarrow[0,1]$ which we know that it is chaotic because "period-3 implies chaos" [34]. We can also consider Figure 6(b) by the above method. Chaos implies that rules 58 have infinite period orbits on its subsystems.

In recent years, associative memory was researched in many papers $[12,13]$. It is obvious that rule 58 can be used in associative memory. By strongly connected graph of rule 58 , we can know the elements on its attractors. Then, we can choose a bit string which belongs to an attractor as memory pattern. Since there are infinite orbits, the storage capability is very large. For example, we can choose 010101 as a memory pattern in Figure 4(c). The associative memory model provides a solution to problem where time to recognize a pattern is independent of the number of patterns stored.

\section{Conclusions}

In this paper, the dynamical behaviors of rule 58 in cellular automata, which is Bernoulli $\sigma_{\tau}$-shift rule, are carefully investigated from viewpoint of symbolic dynamics. We derive the conditions according to Bernoulli $\sigma_{\tau}$-shift evolution for rule 58 . Then, in terms of the transition matrices of determinative block systems of subsystems of rule 58 , we obtain the values of topological entropy of subsystems. According to corresponding strongly connected graph of transition matrices of determinative block systems of subsystems $\Lambda_{1}^{58}$, we divide determinative block systems into two subsets. Then, we find that rule 58 is topologically mixing on $\Lambda_{\lambda_{1}^{58} \text {. Furthermore, we }}$ find that $\left.f_{58}\right|_{\Lambda_{2}^{58}}$ is topologically mixing. So, rule 58 is chaotic in the sense of both Li-Yorke and Devaney. Then, we prove that four rules belonging to global equivalence class $\varepsilon_{4}^{3}$ of $\mathrm{CA}$ are topologically conjugate. We use diagrams to explain the attractors of rule 58, where characteristic function and the Lameray diagram are used to describe that some points fall into Bernoulli-shift map after several times iterations and to show clearly the iterative process of an attractor, respectively. 


\section{Appendix}

Proof of Proposition 1.

Necessity. Suppose that there exists a subset $\Lambda_{1}^{58} \in S^{Z}$ such that $\left.f_{58}\right|_{\Lambda_{1}^{58}}=\left.v\right|_{\Lambda_{1}^{58}}$. Then, $\forall x=\left(\ldots, x_{-1}, x_{0}, x_{1}, \ldots\right) \in \Lambda_{1}^{58}$, we have $\left[f_{58}(x)\right]_{i}=x_{i+1}, \forall x \in Z$.

(1) If $x_{i}=1$, then $x_{i-1} \bar{x}_{i} \oplus \bar{x}_{i-1} x_{i+1}=0 \oplus \bar{x}_{i-1} x_{i+1}$; according to Table 1 , we get $x_{i-1}=0, x_{i+1}=0$, $x_{i+2}=1 ; x_{i-1}=0, x_{i+1}=1$, and $x_{i+2}=0 ; x_{i-2}=0$, $x_{i-1}=1, x_{i+1}=0, x_{i+2}=1$.

(2) If $x_{i}=0$, then $x_{i-1} \bar{x}_{i} \oplus \bar{x}_{i-1} x_{i+1}=x_{i-1} \oplus \bar{x}_{i-1} x_{i+1}$; according to Table 1 , we get $x_{i-2}=0, x_{i-1}=0, x_{i+1}=$ $0 ; x_{i-2}=0, x_{i-1}=0$, and $x_{i+1}=1 ; x_{i-1}=1$, and $x_{i+1}=1$.

(3) Now, we prove that (001) $k x, x \in \Lambda_{1}^{58}$. The proof is by contradiction. Suppose that $(001) \prec x, x \in \Lambda_{1}^{58}$. We can check that $y \in \Lambda_{1}^{58}$, where $f(y)=x$ and $x=\left(\ldots, x_{-3}, x_{-2}, 0, \stackrel{*}{0}, 1, x_{2}, x_{3}, \ldots\right)$. The following is considered in Table 1.

(1) Let $y=\left(y_{-k}, \ldots, y_{-3}, 0,0, \stackrel{*}{0}, 0,1, y_{3}, \ldots, y_{k}\right)$. Firstly, $y_{-3}=0, y_{-4}=0, \ldots y_{-n}=0, \ldots$; otherwise, there exists $(100) \prec y$ which does not belong to $\Lambda_{1}^{58}$. Secondly, there certainly exists $\left(y_{i} y_{i+1} y_{i+2}\right)=(100) \prec$ $y$, where $3 \leq i \leq k$.

(2) Let $y=\left(y_{-k}, \ldots, y_{-3}, y_{-2}, 0,1,0, y_{2}, y_{3}, \ldots, y_{k}\right)$. We cannot get $f\left(y_{-2} 01\right)=0$ in $\Lambda_{1}^{58}$.

(3) Let $y=\left(y_{-k}, \ldots, y_{-3}, y_{-2}, 1,1,0, y_{2}, y_{3}, \ldots, y_{k}\right)$. We cannot get $f\left(y_{-2} 11\right)=0$ in $\Lambda_{1}^{58}$.

Based on the above analysis, these arrive at contradictions.

Therefore, we get that $x_{i-1}, x_{i}$, and $x_{i+1}$ have the following relations:

(i) if $x_{i}=1$, then $x_{i-2}=1, x_{i-1}=0, x_{i+1}=0, x_{i+2}=1$; $x_{i-2}=0, x_{i-1}=0, x_{i+1}=1$, and $x_{i+2}=0 ; x_{i-2}=0$, $x_{i-1}=1, x_{i+1}=0$, and $x_{i+2}=1$.

(ii) If $x_{i}=0$, then $x_{i-2}=0, x_{i-1}=0, x_{i+1}=0$, and $x_{i+2}=0 ; x_{i-1}=1$ and $x_{i+1}=1$.

Sufficiency. Suppose that there exists a subset $\Lambda_{1}^{58} \subset S^{Z}$, and, $\forall x \in \Lambda_{1}^{58}$, the relations between $x_{i-1}, x_{i}$, and $x_{i+1}$ satisfy the conditions (i) and (ii) in Proposition $1, \forall i \in Z$.

(i) If $x_{i}=1$, we have $\left[f_{58}(x)\right]_{i}=x_{i-1} \bar{x}_{i} \oplus \bar{x}_{i-1} x_{i+1}=$ $0 \oplus \bar{x}_{i-1} x_{i+1}$.

Therefore,

$$
\left[f_{58}(x)\right]_{i}=\left\{\begin{array}{lll}
0, & x_{i-1}=0, & x_{i+1}=0 \\
1, & x_{i-1}=0, & x_{i+1}=1, \\
0, & x_{i-1}=1, & x_{i+1}=0 .
\end{array}\right.
$$

(ii) If $x_{i}=0$, we have $\left[f_{58}(x)\right]_{i}=x_{i-1} \bar{x}_{i} \oplus \bar{x}_{i-1} x_{i+1}=$ $x_{i-1} \oplus \bar{x}_{i-1} x_{i+1}$.

Therefore,

$$
\left[f_{58}(x)\right]_{i}=\left\{\begin{array}{lll}
0, & x_{i-1}=0, & x_{i+1}=0 \\
1, & x_{i-1}=1, & x_{i+1}=1
\end{array}\right.
$$

Hence, $\left[f_{58}(x)\right]_{i}=x_{i+1}$.

Proof of Proposition 17. (a) Let any two open sets $U, V \subset \Lambda_{2}^{58}$. By Proposition 13 (b), we know that $\left.f_{58}^{2}\right|_{\Lambda_{2}^{58}}$ is topologically mixing; then there exists $N_{1}>0$, such that $\left(\left.f_{58}^{2}\right|_{\Lambda^{58}}\right)^{n}(U) \cap$ $V \neq \emptyset, \forall n \geq N_{1}$. Then we consider the following two cases.

Case 1. Consider $n=2 k, k \in Z^{+}$; then

$$
\begin{aligned}
& \left(\left.f_{58}\right|_{\Lambda_{2}^{58}}\right)^{n}(U) \cap V \\
& \quad=\left(\left.f_{58}\right|_{\Lambda_{2}^{58}}\right)^{2 k}(U) \cap V \\
& =\left(\left.f_{58}^{2}\right|_{\Lambda_{2}^{58}}\right)^{k}(U) \cap V \neq \emptyset, \quad \forall n \geq 2 N_{1} .
\end{aligned}
$$

Case 2. Consider $n=2 k+1, k \in Z^{+}$. It is obvious that $\Lambda_{2}^{58}$ is surjective. Suppose that there exist $y, y_{1} \in \Lambda_{2}^{58}$ such that $f_{58}(y)=f_{58}\left(y_{1}\right)$. Thus, $f_{58}^{2}(y)=f_{58}^{2}\left(y_{1}\right)$ holds, which implies $y=y_{1}$. Therefore, $\left.f_{58}\right|_{\Lambda^{58}}$ is injective. Since $\Lambda_{2}^{58}$ is a compact Hausdorff space and $\left.f_{58}\right|_{\Lambda_{2}^{58}}$ is one-to-one onto, and continuous, $\left.f_{58}^{-1}\right|_{\Lambda^{58}}$ exists and is continuous. Therefore, $\left.f_{58}\right|_{\Lambda^{58}}$ is a homeomorphism, which implies that $f_{58}(U)$ is an open set. Thus,

$$
\begin{aligned}
& \left(\left.f_{58}\right|_{\Lambda_{2}^{58}}\right)^{n}(U) \cap V \\
& \quad=\left(\left.f_{58}\right|_{\Lambda_{2}^{58}}\right)^{2 k} \circ f_{58}(U) \cap V \neq \emptyset, \quad \forall n \geq 2 N_{1}+1 .
\end{aligned}
$$

Hence, for any two open sets $U, V, \exists N=2 N_{1}+1$, such that $\left(\left.f_{58}\right|_{\Lambda_{2}^{58}}\right)^{n}(U) \cap V \neq \emptyset, \forall n \geq N$; namely, $\left.f_{58}\right|_{\Lambda_{2}^{58}}$ is topologically mixing.

(b) Consider $\operatorname{ent}\left(\left.f_{58}\right|_{\Lambda_{2}^{58}}\right)=(1 / 2) \operatorname{ent}\left(\left.f_{58}^{2}\right|_{\Lambda_{2}^{58}}\right)=0.2812$.

\section{Conflict of Interests}

The authors declare that there is no conflict of interests regarding the publication of this paper.

\section{Acknowledgments}

This work was supported in part by Research Project of Chongqing University of Science and Technology 
(CK2013B15), in part by Scientific and Technological Research Program of Chongqing Municipal Education Commission (Grants nos. KJ131401 and KJ131416), in part by Natural Science Foundation Project of CQ CSTC (Grants nos. cstc2012jjB0095 and cstc2012jjA1459), in part by the National Natural Science Foundation of China (51275547), in part by Achievement Transfer Program of Institutions of Higher Education in Chongqing (KJ121413), and in part by Teaching \& Research Program of Chongqing University of Science and Technology (X2012-29-S).

\section{References}

[1] J. Neumann, The General and Logical Theory of Automata, Pergamon Press, London, UK, 1951.

[2] H. Beigy and M. R. Meybodi, "Cellular learning automata with multiple learning automata in each cell and its applications," IEEE Transactions on Systems, Man, and Cybernetics B: Cybernetics, vol. 40, no. 1, pp. 54-65, 2010.

[3] W. Pries, A. Thanailakis, and H. C. Card, "Group properties of cellular automata and VLSI applications," IEEE Transactions on Computers C, vol. 35, no. 12, pp. 1013-1024, 1986.

[4] K. M. Faraoun, "Design of fast one-pass authenticated and randomized encryption schema using reversible cellular automata," Communications in Nonlinear Science and Numerical Simulation, vol. 19, no. 9, pp. 3136-3148, 2014.

[5] P. Ping, F. Xu, and Z. Wang, "Color image encryption based on two-dimensional cellular automata," International Journal of Modern Physics C, vol. 24, no. 10, Article ID 1350071, 14 pages, 2013.

[6] S. Nandi, B. K. Kar, and P. Pal Chaudhuri, "Theory and applications of cellular automata in cryptography," IEEE Transactions on Computers, vol. 43, no. 12, pp. 1346-1357, 1994.

[7] M. Tomassini and M. Perrenoud, "Cryptography with cellular automata," Applied Soft Computing, vol. 1, no. 2, pp. 151-160, 2001.

[8] J.-C. Jeon and K.-Y. Yoo, "Elliptic curve based hardware architecture using cellular automata," Mathematics and Computers in Simulation, vol. 79, no. 4, pp. 1197-1203, 2008.

[9] J. Machicao, A. G. Marco, and O. M. Bruno, "Chaotic encryption method based on life-like cellular automata," Expert Systems with Applications, vol. 39, no. 16, pp. 12626-12635, 2012.

[10] Z. Eslami, S. H. Razzaghi, and J. Z. Ahmadabadi, "Secret image sharing based on cellular automata and steganography," Pattern Recognition, vol. 43, no. 1, pp. 397-404, 2010.

[11] R. J. Chen and S. J. Horng, "Novel SCAN-CA-based image security system using SCAN and 2-D von Neumann cellular automata," Signal Processing: Image Communication, vol. 25, no. 6, pp. 413-426, 2010.

[12] P. Maji and P. Pal Chaudhuri, "Non-uniform cellular automata based associative memory: evolutionary design and basins of attraction," Information Sciences, vol. 178, no. 10, pp. 2315-2336, 2008.

[13] N. Ganguly, P. Maji, B. K. Sikdar, and P. P. Chaudhuri, "Design and characterization of cellular automata based associative memory for pattern recognition," IEEE Transactions on Systems, Man, and Cybernetics B, vol. 34, no. 1, pp. 672-679, 2004.

[14] S. Wolfram, "Universality and complexity in cellular automata," Physica D: Nonlinear Phenomena, vol. 10, no. 1-2, pp. 1-35, 1984.

[15] S. Wolfram, Theory and Application of Cellular Automata, World Scientific, Singapore, 1986.
[16] S. Wolfram, A New Kind of Science, Wolfram Media, Champaign, Ill, USA, 2002.

[17] L. O. Chua, S. Yoon, and R. Dogaru, "A nonlinear dynamics perspective of Wolfram's new kind of science. I. Threshold of complexity," International Journal of Bifurcation and Chaos in Applied Sciences and Engineering, vol. 12, no. 12, pp. 2655-2766, 2002.

[18] L. O. Chua, V. I. Sbitnev, and S. Yoon, "A nonlinear dynamics perspective of Wolfram's new kind of science, part II: universal neuron," International Journal of Bifurcation and Chaos in Applied Sciences and Engineering, vol. 13, no. 9, pp. 2377-2491, 2003.

[19] L. O. Chua, V. I. Sbitnev, and S. Yoon, "A nonlinear dynamics perspective of Wolfram's new kind of science. III. Predicting the unpredictable," International Journal of Bifurcation and Chaos in Applied Sciences and Engineering, vol. 14, no. 11, pp. 3689-3820, 2004.

[20] L. O. Chua, V. I. Sbitnev, and S. Yoon, "A nonlinear dynamics perspective of Wolfram's new kind of science, part IV: from Bernoulli shift to $1 / f$ spectrum," International Journal of Bifurcation and Chaos in Applied Sciences and Engineering, vol. 15, no. 4, pp. 1045-1183, 2005.

[21] L. O. Chua, V. I. Sbitnev, and S. Yoon, "A nonlinear dynamics perspective of Wolfram's new kind of science. V. Fractals everywhere," International Journal of Bifurcation and Chaos in Applied Sciences and Engineering, vol. 15, no. 12, pp. 3701-3849, 2005.

[22] L. O. Chua, V. I. Sbitnev, and S. Yoon, "A nonlinear dynamics perspective of Wolfram's new kind of science, part VI: from time-reversible attractors to the arrow of time," International Journal of Bifurcation and Chaos, vol. 16, no. 5, pp. 1097-1373, 2006.

[23] F. Y. Chen, W. F. Jin, G. R. Chen, F. F. Chen, and L. Chen, "Chaos of elementary cellular automata rule 42 of Wolfram's class II," Chaos, vol. 19, no. 1, Article ID 013140, 2009.

[24] F. F. Chen and F. Y. Chen, "Complex dynamics of cellular automata rule 119," Physica A: Statistical Mechanics and its Applications, vol. 388, no. 6, pp. 984-990, 2009.

[25] L. Chen, F. Y. Chen, and W. Jin, "Complex symbolic dynamics of bernoulli shift cellular automata rule," in Proceedings of the 9th International Conference for Young Computer Scientists (ICYCS '08), pp. 2868-2873, Hunan, China, November 2008.

[26] W. Jin, F. Chen, G. Chen, L. Chen, and F. Chen, "Extending the symbolic dynamics of Chua's Bernoulli-shift rule 56," Journal of Cellular Automata, vol. 5, no. 1-2, pp. 121-138, 2010.

[27] L. Chen, F. Chen, W. Jin, and G. Chen, "Some nonrobust Bernoulli-shift rules," International Journal of Bifurcation and Chaos, vol. 19, no. 10, pp. 3407-3415, 2009.

[28] Q. Han, X. F. Liao, C. D. Li, and L. P. Feng, "Complex dynamics behaviors in cellular automata rule 35," Journal of Cellular Automata, vol. 6, no. 6, pp. 487-504, 2011.

[29] Q. Han, X. F. Liao, and C. D. Li, "Complex dynamic behaviors in cellular automata rule 14," Discrete Dynamics in Nature and Society, vol. 2012, Article ID 258309, 12 pages, 2012.

[30] J. Guan, S. Shen, C. Tang, and F. Chen, "Extending Chua's global equivalence theorem on Wolfram's new kind of science," International Journal of Bifurcation and Chaos, vol. 17, no. 12, pp. 4245-4259, 2007.

[31] B. Kitchens, Symbolic Dynamics: One-sided, Two-sided and Countable State Markov Shifts, Springer, Berlin, Germany, 1990. 
[32] Z. Zhou, Symbolic Dynamics, Shanghai Scientific and Technological Education Publishing House, Shanghai, China, 1997, (Chinese).

[33] L. P. Shilnikov, A. L. Shilnikov, D. V. Turaev, and L. O. Chua, Methods of Qualitative Theory in Nonlinear Dynamics, World Scientific, Singapore, 1998.

[34] T. Y. Li and J. A. Yorke, "Period three implies chaos," The American Mathematical Monthly, vol. 82, no. 10, pp. 985-992, 1975. 


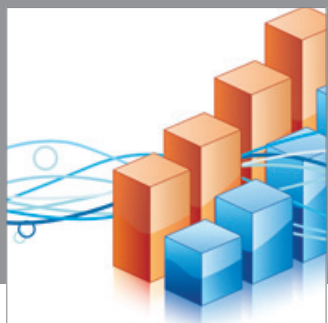

Advances in

Operations Research

mansans

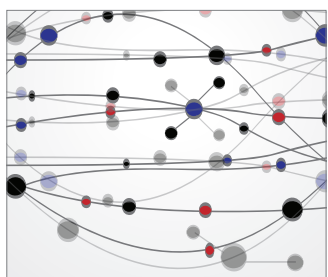

The Scientific World Journal
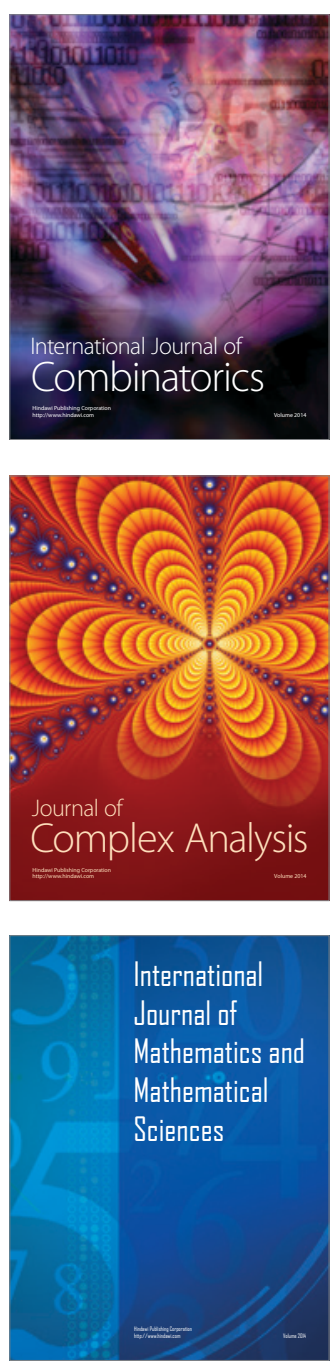
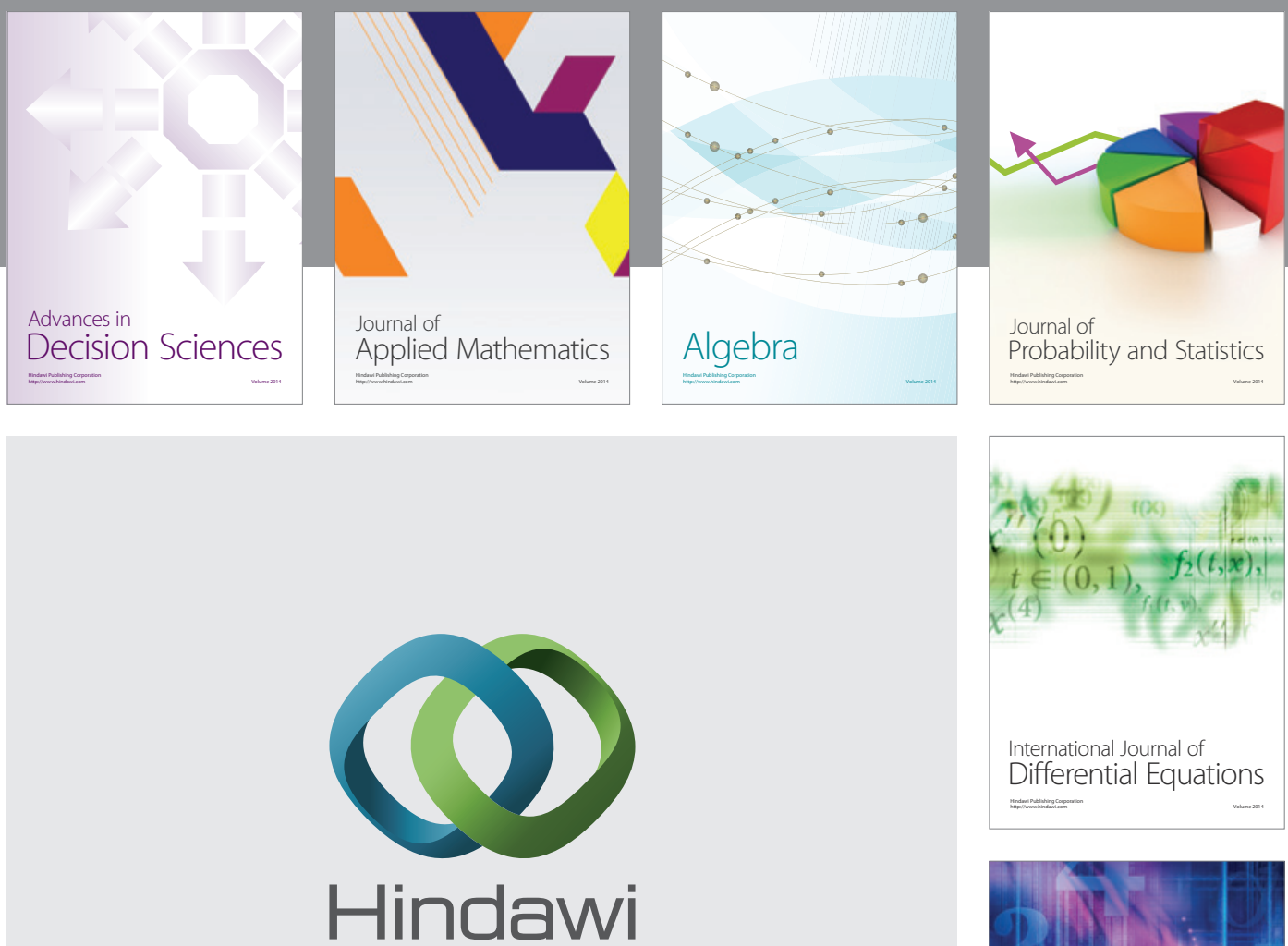

Submit your manuscripts at http://www.hindawi.com
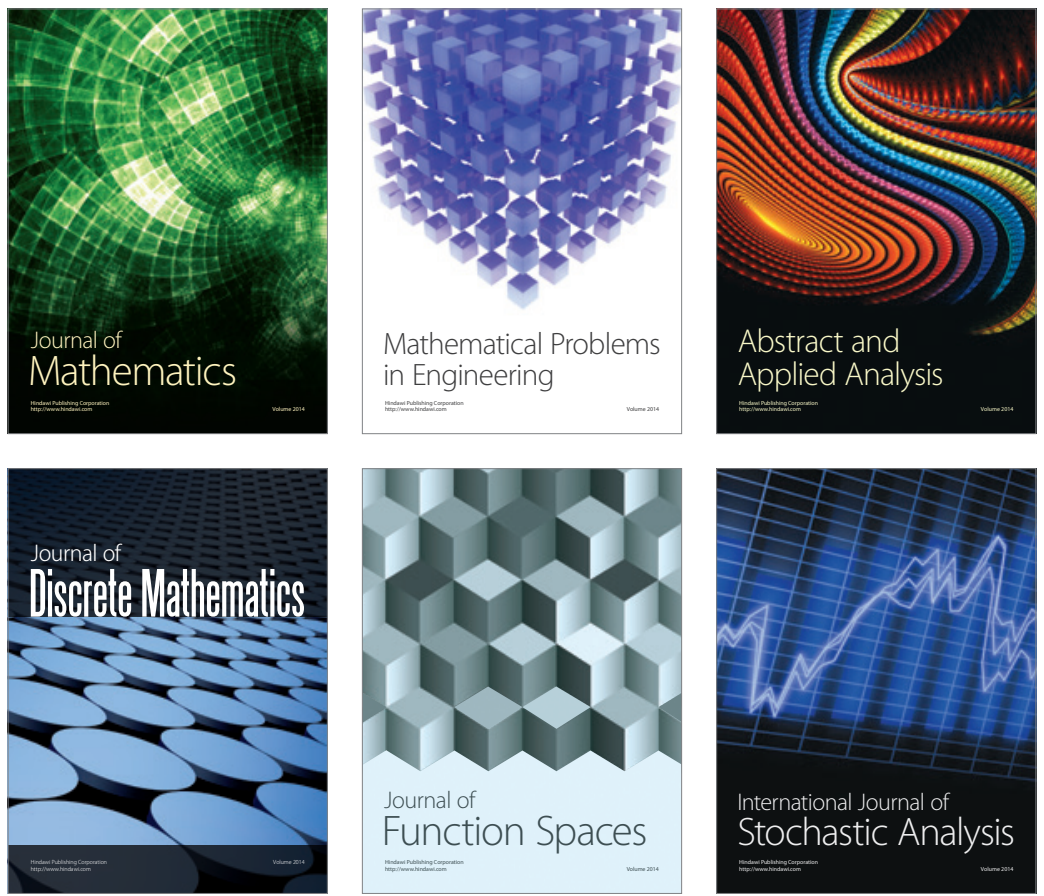

Journal of

Function Spaces

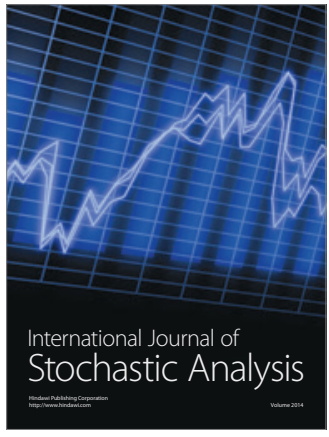

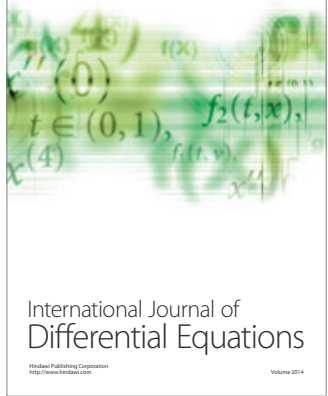
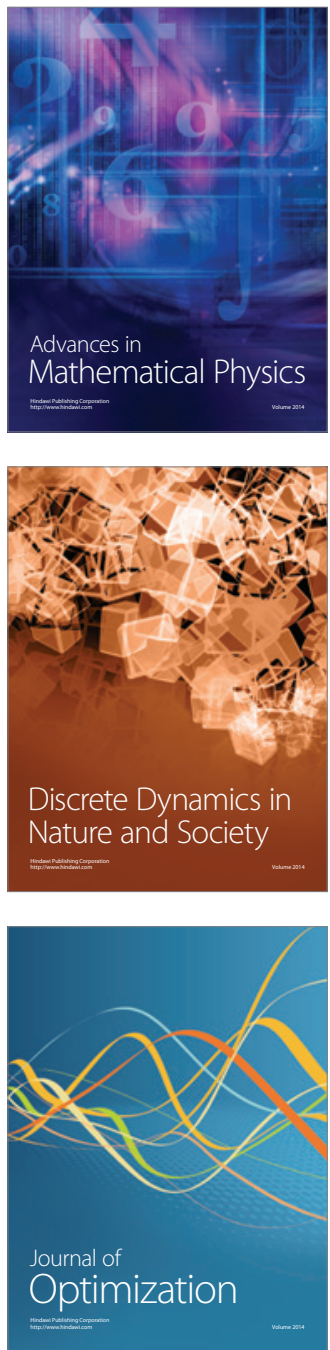